\title{
Correction to: Retrospective dosimetry study of intensity-modulated radiation therapy for nasopharyngeal carcinoma: measurement-guided dose reconstruction and analysis
}

Wen-zhao Sun ${ }^{\dagger}$, Dan-dan Zhang ${ }^{\dagger}$, Ying-lin Peng, Li Chen, De-hua Kang, Bin Wang and Xiao-wu Deng*

\section{Correction}

The original version of this article [1] unfortunately contained a mistake. The presentation of Table 4 was incorrect. The corrected table is given below and the original article was corrected.

Received: 23 March 2018 Accepted: 7 June 2018

Published online: 25 June 2018

\section{Reference}

1. Sun WZ, et al. Retrospective dosimetry study of intensity-modulated radiation

therapy for nasophanyngeal carcinoma: measurement-guided dose reconstruction and analysis. Radiat Oncol. 2018;13:42. https:/doi.org/10.1186/s13014-018-0993-2

* Correspondence: dengxw@mail.sysu.edu.cn

'Wen-zhao Sun and Dan-dan Zhang contributed equally to this work.

State Key Laboratory of Oncology in South China, Collaborative Innovation Center for Cancer Medicine, Department of Radiation Oncology, Sun Yat-sen University Cancer Center, 651 Dongfeng Road East, Guangzhou 510060,

People's Republic of China 
Table 4 Pearson correlation coefficient with three type gamma pass rate and $D_{V}, V_{D}$

\begin{tabular}{|c|c|c|c|c|c|c|c|c|c|c|}
\hline \multirow[t]{2}{*}{ Structure } & \multirow{2}{*}{$\begin{array}{l}\text { DVH } \\
\text { index }\end{array}$} & \multicolumn{3}{|c|}{ Planar 2D GP(\%) } & \multicolumn{3}{|c|}{ Global 3D GP(\%) } & \multicolumn{3}{|c|}{ Organ specific 3D GP(\%) } \\
\hline & & $3 \mathrm{~mm} / 3 \%$ & $2 \mathrm{~mm} / 2 \%$ & $1 \mathrm{~mm} / 1 \%$ & $3 \mathrm{~mm} / 3 \%$ & $2 \mathrm{~mm} / 2 \%$ & $1 \mathrm{~mm} / 1 \%$ & $3 \mathrm{~mm} / 3 \%$ & $2 \mathrm{~mm} / 2 \%$ & $1 \mathrm{~mm} / 1 \%$ \\
\hline \multirow[t]{4}{*}{ PTVnx } & V100\% & -0.038 & -0.106 & -0.252 & 0.044 & -0.145 & -0.238 & -0.328 & -0.405 & -0.267 \\
\hline & D98\% & -0.143 & -0.099 & -0.217 & 0.010 & -0.244 & -0.391 & -0.358 & -0.425 & -0.291 \\
\hline & $\mathrm{D} 2 \%$ & -0.336 & -0.332 & -0.460 & -0.336 & -0.398 & -0.409 & -0.754 & $-0.810^{*}$ & $-0.811^{*}$ \\
\hline & Dmean & -0.386 & -0.409 & -0.585 & -0.248 & -0.442 & -0.567 & $-0.836^{*}$ & $-0.968^{*}$ & $-0.935^{*}$ \\
\hline \multirow[t]{2}{*}{ PTV1 } & V100\% & -0.035 & -0.217 & -0.210 & -0.174 & -0.232 & -0.258 & -0.166 & -0.189 & 0.056 \\
\hline & D95\% & 0.131 & 0.065 & -0.110 & 0.162 & -0.124 & -0.362 & -0.135 & -0.409 & -0.431 \\
\hline \multirow[t]{2}{*}{ PTV2 } & V100\% & 0.046 & 0.152 & 0.118 & -0.057 & -0.164 & -0.151 & -0.072 & -0.102 & -0.006 \\
\hline & D95\% & -0.168 & -0.253 & -0.225 & -0.563 & -0.58 & -0.467 & -0.525 & -0.458 & -0.376 \\
\hline SC & D1cc & -0.185 & -0.157 & -0.216 & -0.309 & -0.478 & -0.443 & -0.595 & -0.664 & -0.650 \\
\hline BS & V60 Gy & -0.147 & -0.260 & -0.118 & -0.111 & -0.376 & -0.421 & -0.140 & -0.384 & -0.474 \\
\hline \multirow[t]{2}{*}{ Parotid-L } & Dmean & 0.160 & 0.079 & 0.004 & 0.118 & 0.042 & -0.050 & -0.007 & -0.173 & -0.385 \\
\hline & V30 Gy & 0.297 & 0.096 & -0.115 & 0.158 & 0.017 & -0.122 & 0.065 & -0.377 & -0.403 \\
\hline \multirow[t]{2}{*}{ Parotid-R } & Dmean & -0.056 & -0.022 & -0.049 & 0.091 & -0.034 & -0.151 & 0.007 & -0.126 & -0.489 \\
\hline & V30 Gy & -0.064 & 0.056 & -0.057 & 0.093 & -0.085 & -0.207 & -0.057 & -0.147 & -0.42 \\
\hline TL-L & D1cc & 0.043 & 0.146 & 0.139 & 0.151 & 0.132 & 0.087 & 0.068 & -0.096 & -0.271 \\
\hline$T L-R$ & D1cc & 0.055 & 0.162 & 0.196 & 0.058 & 0.035 & 0.050 & 0.370 & -0.164 & -0.241 \\
\hline Larynx & Dmean & -0.225 & -0.288 & -0.243 & -0.315 & -0.366 & -0.363 & 0.079 & -0.349 & -0.666 \\
\hline
\end{tabular}

The two-tailed Student t-test with SPSS statistical software (V19);

Bold letters in the data highlighted $R>0.8$ and ${ }^{*}$ symbolled the significance of the correlation at the 0.01 level $(P<0.01$, bilateral);

Abbreviations: $D_{V}$ volumetric dose, $V_{D}$ dose volumes, $P T V$ planning target volume, GP (\%) gamma pass rate (\%), SC spinal cord, BS brainstem, Parotid-L left parotid gland, Parotid- $R$ right parotid gland, $T L-L$ left temporal lobe, $T L-R$ right temporal lobe 